\title{
Comparative Study of Different Assisted Hatching Techniques and In Vitro Culture Media on Mice Embryo Hatching Rate
}

Negota NC ${ }^{1}$ Mphaphathi $\mathrm{ML}^{3}$, Nethenzheni LP$^{1}$, Rammutla TL ${ }^{1}$, Serota NR ${ }^{1}$, Barry $\mathrm{DM}^{1}$ and Nedambale TL $^{2,3 *}$

${ }^{1}$ Department of Animal science, University of Venda, South Africa

${ }^{2}$ Department of Animal Science, Tshwane University of Technology, South Africa

${ }^{3}$ Germplasm Conservation and Reproductive Biotechnology, Agricultural Research Council, South Africa

*Corresponding author: Nedambale TL, Department of Animal Science, Tshwane University of Technology, Germplasm Conservation \& Reproductive Biotechnology, Private Bag X680, Pretoria, 0001, South Africa

Received: January 25, 2021; Accepted: February 17, 2021; Published: February 24, 2021

\begin{abstract}
The study investigated the influence of Assisted Hatching (AH) techniques (mechanical, chemical, enzymatic thinning and laser) and two in vitro culture media (Ham's F10 and TCM-199) on hatching rate of mice embryos (blastocyststage) following 24 hours of culture. The C57BL/6-black $(n=30)$ and BALB/cwhite $(n=30)$ mouse breeds were raised until maturity and naturally bred to produce a F1 generation. The light in the breeding house was controlled and the mice were fed ad libitum. Female mice $(n=30)$ were injected (peritoneal) with $0.1 \mathrm{ml}(5 \mathrm{IU})$ of eCG into the abdominal cavity with $1 \mathrm{ml}$ syringe and $0.5 \times 16 \mathrm{~mm}$ needle to stimulate follicular growth and $46-49 \mathrm{~h}$ later was injected with $0.1 \mathrm{ml}(5 \mathrm{IU})$ of hCG to cause ovulation, maintain the corpus luteum and stimulate it to secrete progesterone for maintenance of pregnancy. After the injection, the male and female (1:1) could mate overnight. Female mice with vaginal plugs were observed and kept separately for blastocyst-stage embryos collection on day three following successful mating. They were euthanized, and abdomen sterilized with $70 \%$ ethanol. Using a sterile surgical scissor, a fine cut was made, holding the skin firmly above and below the incision, the skin was pulled apart using forceps. The embryos were flushed from the uterus using a 30-gauge needle with culture media. Following the AH techniques, embryos were cultured in TCM-199 or Ham's F10 for 24 hours and zonal thickness of all hatched embryos were measured. Immediately after assisted hatching, the embryos were cultured into two different in vitro culture media. All embryos hatched were stained and the zonal thickness of embryos were measured. The number of blastomeres were counted and recorded $24 \mathrm{~h}$ later. Data collected were subjected to analysis of variance using PROC General Linear Model. The Tukey's test was used to separate the means. A significant difference was observed between the thickness of Zona Pellucida (ZP) pre and post treatment after 24 hours of culture. However, there was no significance difference among blastocyst hatching rate and the blastomeres nuclei counted after staining. The thickness of the ZP decreased with individual $\mathrm{AH}$ techniques. The interaction between $\mathrm{AH}$ techniques and in vitro culture was found to be significantly different on blastocyst hatchability. However, laser $\mathrm{AH}$ technique had highest hatchability (56.3\%) when embryos were cultured in TCM-199 followed by mechanical AH techniques $(52.6 \%)$. The hatchability rate $(33.3 \%)$ was recorded in the chemical $\mathrm{AH}$ technique group. The blastomeres nuclei counted under interaction of $\mathrm{AH}$ techniques and culture media was not differently affected, with the values ranging from 69 to $76 \%$. In conclusion, the use of different $\mathrm{AH}$ techniques resulted in varying effect and increase outcomes towards the hatching rate.
\end{abstract}

Keywords: Blastocyst; Assisted Hatching; Mouse; Zona Pellucida; Ham's F10; TCM-199

\section{Introduction}

Biologically, after an embryo has reached the blastocyst-stage, the Zona Pellucida (ZP), becomes thinner and groove appears allowing blastocyst to hatch and implants to the uterine wall. The hatching of the blastocyst is the crucial step that must take place before implantation. The failure of $\mathrm{ZP}$ rapture impairs the hatching of the blastocyst, thus limiting conception rate [1]. Assisted Hatching (AH) is one of the assisted reproductive technology methods that artificially manipulates the ZP. The use of AH methods has been reported to be essential for improving implantation rates of embryos in both animals and humans [2].The use of different $\mathrm{AH}$ techniques to create a hole in the ZP or weaken the ZP at the blastocyst-stage of embryo development has been previously documented [3]. Furthermore, it allows easy hatching of the blastocyst from the ZP $[3,4]$ recorded an improvement in pregnancy rate after the use of laser $\mathrm{AH}$ technique in embryos. Assisted hatching can be done by a variety of techniques, including the use of chemical, mechanical, enzymatic and laser assisted hatching techniques $[5,6]$ reported that $\mathrm{AH}$ techniques addresses the challenge of embryo hatching from the ZP by improving the potential
J Bacteriol Mycol - Volume 8 Issue 1 - 2021 ISSN : 2471-0172 | www.austinpublishing group.com Nedambale et al. @ All rights are reserved
Citation: Negota NC, Mphaphathi ML, Nethenzheni LP, Rammutla TL, Serota NR, Barry DM, et al. Comparative Study of Different Assisted Hatching Techniques and In Vitro Culture Media on Mice Embryo Hatching Rate. J Bacteriol Mycol. 2021; 8(1): 1163 
of blastocyst-stage embryos to hatch in vitro.

Mechanical AH technique is done by dissection and creating an opening in the ZP of the blastocyst, while the embryo is held stable with a holding pipette. The $\mathrm{ZP}$ is then pierced with a micro needle through the space between the $\mathrm{ZP}$ and blastomeres. Acidified tyrode's solution is used for the chemical $\mathrm{AH}$ technique and it has been previously reported in detail by [6]. The enzymatic mechanism is enzymatic digestion of the $\mathrm{ZP}$ using trypsin enzyme. The laser $\mathrm{AH}$ technique uses a laser beam to drill the outer part of the ZP and can be done with high precision and repeatability. It is reported that there is no side effect on embryo development. These techniques have been used, but there is information on which of the method is the most effective when used with which type of in vitro culture media. It was reported that $\mathrm{AH}$ can exert both a beneficial as well as a deleterious effect, on subsequent embryonic development depending on several factors. These factors include, ZP thickness, the size of the hole created, mechanical damage to the embryo by manipulation, chemical damage by acid solution and the technical skill of the operator [7].

The ZP is layer composed of glycoprotein, carbohydrates and zona pellucida-specific proteins that cover the oocytes before fertilization until hatching of the embryo. Before hatching, the blastocyst should undergo a series of contractions and expansions that cause a decrease in the thickness of the ZP until it becomes almost invisible [8]. But this process is hindered by the increase in the ZP thickness of the embryo which in turn affect the process of embryo hatching [9]. The hardening or thickening of the ZP after fertilization was proposed as the one that limit hatching rate in mammalian embryos [10].

Failure to hatch has been largely attributed to ZP hardening and suboptimal culture conditions. In vitro produced embryos may result in hardening of the ZP that is resistant to thinning and subsequently impair the hatching process, whereas, in vivo produced embryos may result in loss of uniformity and thinning of ZP, which precede to hatching. Sub-sequential culture media are also used to increase blastulation and pregnancy rates in assisted reproduction treatments.

In vitro processes involve the use of culture media, which is essential in the maturation and development of embryos. The constituents of the culture medium have got an effect on the cultured embryo development. Biotechnology laboratories are using lot of different in vitro culture media and the selection of good, reliable and clean media is a necessity for their activities [11]. Reported on the use of TCM-199 that showed an increase in blastocyst formation of embryos during in vitro culture. There are differences in the degree to which these media support embryos development. However, the reviewed information concerning (TCM-199 and Ham's F10) is that they are all good enhancers of high fertilization [12]. Authors hypothesized that $\mathrm{AH}$ can enhance hatching rate of the blastocyststage embryos, by both catalyzing the time and rate of hatching. Therefore, the present study was conducted to compare four $\mathrm{AH}$ techniques (mechanical, chemical, enzymatic thinning and laser) and use of two in vitro culture media (Ham's F10 and TCM-199) on hatching rate of mice embryos (blastocyst-stage) following 24 hours in vitro of embryos in different groups per droplet.

\section{Materials and Methods}

\section{Study site}

The study was conducted at the Centre of Excellence in
Assisted Animal Reproduction, Biotechnology Laboratory, School of Agriculture, University of Venda, Thohoyandou, Limpopo, South Africa. Coordinates are as follow (22 58' S, $30^{\circ} 26^{\prime}$ E). Daily temperatures at Thohoyandou vary from about $25^{\circ} \mathrm{C}$ to $40^{\circ} \mathrm{C}$ in summer and between approximately $12^{\circ} \mathrm{C}$ and $26^{\circ} \mathrm{C}$ in winter. Rainfall is highly seasonal with $95 \%$ occurring between October and March. The average rainfall is about $800 \mathrm{~mm}$ but varies [13].

\section{Chemicals}

The culture media used in this study were Ham's F10 and TCM199 (Sigma). The hormones were eCG (Sigma), hCG (Sigma), Ham's F10, TCM-199 and Tyrodes solution (Sigma).

\section{Experimental design}

Three factors were investigated. These were $\mathrm{AH}$ techniques and in vitro culture medium culture. The first factor involved four $\mathrm{AH}$ techniques; Number of AH (control), laser, mechanical, chemical, and enzymatic. The second factor involve in vitro culture media; TCM-199 and Ham's F10. The third factor involve embryo grouping per $50 \mu \mathrm{l}$ droplet; one, two, three and four embryos per drop. The experiment was set up as $4 \times 2 \times 4$ factorial design arranged in a Completely Randomized Design (CRD) to determine the significant differences.

\section{Management of mouse}

Mice were housed in cages with sawdust. They were raised and fed with mouse pellets and water ad libitum. The sawdust in the cages was changed every 5 days. The light in the breeder house was regulated day and night. The light was on from 5 am until 7 pm (14 hour) and was switched off from 7 pm until 5 am (10 hour). A constant temperature of $24^{\circ} \mathrm{C}$ was maintained in the breeder house.

\section{Production of F1 generation}

Black female mice (C57BL/6) were mated with white male mice $(\mathrm{BALB} / \mathrm{c})$ and were kept together in the breeding cage for 7 days. After 7 days the males were removed and returned to their original cages. The F1 offspring were born 21 days after mating. The offspring were raised until they were 6 weeks old.

\section{Preparation and Administering of Injections for $\mathbf{F} 1$}

\section{Folligon}

Folligon is available in a bottle of 1000 International Units (IU) each (Folligon, Intervet SA, MSD Animal Health). One bottle of $1000 \mathrm{IU}$ was diluted with saline using a syringe to give a working concentration of $50 \mathrm{IU} / \mathrm{ml}=5 \mathrm{IU} / 0.1 \mathrm{ml}$. The solution was stored as $5 \mathrm{IU}$ in $1.0 \mathrm{ml}$ syringes and kept frozen at $-30^{\circ} \mathrm{C}$.

\section{Chorulon}

Chorulon is available in a concentration of 1500 (IU) of hCG (Chorulon, Intervet SA, MSD Animal Health). This was dissolved in the saline solvent. The Folligon was brought to a concentration of 50 $\mathrm{IU} / \mathrm{ml}=5 \mathrm{IU} / 0.1 \mathrm{ml}$. The solution was stored as $5 \mathrm{IU}$ in $1.0 \mathrm{ml}$ syringes and kept frozen at $-30^{\circ} \mathrm{C}$.

Female mice $(\mathrm{n}=30)$ were injected (peritoneal) with $0.1 \mathrm{ml}(5 \mathrm{IU})$ of eCG into the abdominal cavity with $1 \mathrm{ml}$ syringe and $0.5 \times 16 \mathrm{~mm}$ needle, and $46-49 \mathrm{~h}$ later, $0.1 \mathrm{ml}$ (5 IU) of hCG was injected. The eCG was used to stimulate follicular growth and hCG was used to mimic 
Luteinizing Hormone (LH) to cause ovulation, maintains the corpus luteum and stimulates it to secrete progesterone for maintenance of pregnancy. After the injections, the F1 male and female mice (ratio of 1:1) could mate overnight and the next morning the vaginal plugs were observed to confirm mating. The mice with vaginal plugs were kept separately for the collection of blastocyst-stage embryos on day three following successful mating.

\section{Blastocyst stage embryo collection}

On day three after successful mating, the mouse was taken from the cage and placed on the cage bars, so that it can grip the bars with its front paws. The cervical vertebra was separated by applying firm pressure at the base of the head (back of the neck). The mouse was laid on its back on the absorbent paper and its abdomen soaked with 70\% ethanol to maintain sterility. A fine cut was made in the middle of the abdomen by using a sterile surgical scissor, while holding the skin firmly above and below the incision. The skin was pulled apart using watchmaker forceps. The blastocyst-stage embryos were collected from the uterine horn by flushing the embryos using the D-PBS loaded in the tip of a pipette inserted onto a $1 \mathrm{ml}$ syringe. A 26-gauge needle was inserted into the tip of the uterine horn (from the ovarian side) to flush blastocyst embryo towards the uterine body. A $1 \mathrm{ml}$ syringe with a pipette tip was used to pick up the blastocyst-stage embryos and washed three times in droplets of D-PBS and culture media as prepared in the tables below.

\section{Mechanical assisted hatching technique (partial zona dissection)}

In brief, the 400 embryos were held by a holding pipette held $30^{\circ}$ from the left and the Partial Zona Dissection (PZD) injection needle was inserted from a right of the embryo. The $\mathrm{ZP}$ of the embryo was pierced with the PZD injection needle with extensive care in order to avoid damage to the inner cell mass. The $\mathrm{ZP}$ was pierced with the needle so that a hole is created on it.

\section{Chemical assisted hatching technique (acidified tyrode's solution)}

A concentration of tyrode's solution with a $\mathrm{pH}$ of $2.5-3$ in $99.875 \%$ of culture medium was used to digest the ZP. The holding pipette was lowered into a drop containing the blastocyst-stage embryos $(n=10)$ to be digested in order to control the movement of embryos. Embryos could rest stable in the petri dish with either TCM-199 or Ham's F10 culture medium. The injection needle was removed from the packet and the tip of the needle was party broken using a hand to make a hole on it that will allow easy aspiration of acidified tyrode's solution inside the injection needle. The injection needle was filled with acidified tyrode's solution. The same injection needle related to the homemade mouth-control delivery system. A volume of $20 \mu \mathrm{l}$ acidified tyrode's solution was aspirated and a mouth-controlled homemade delivery system was used to blow acidified tyrode's solution gradually over a small area of the ZP until it is digested. The embryos were immediately removed and washed two times in the TCM-199 or Ham's F10 culture medium to remove traces of acids.

\section{Laser assisted hatching technique}

The dish containing the mouse blastocyst-stage embryos $(n=10)$ was placed on the stage of the inverted microscope and the embryos were located under focus. The laser lens was rotated to the position of the drops and was focused on the first embryo. The laser target was placed over the ZP in a place that had no blastomeres directly underneath. The laser beam was guided by safety circles which help the operator to choose the area of interest and the target was positioned on the ZP and ensure that the safety circle did not overlap the blastomeres. Beaming of the ZP was done with a single medium pulse on the ZP of the all the embryos, without breaking the layer of blastomeres. The same procedure was repeated for all the embryos in TCM-199 or Ham's F10 medium.

\section{Enzymatic with trypsin assisted hatching techniques}

The TCM-199 or Ham's F10 medium with the concentrations of $0.5 \%$ trypsin, $\mathrm{pH}$ of $6.8-7.2$ and $0.1 \%$ of EDTA was used in the digestion of the ZP. Assisted hatching technique using the above medium was prepared in a $35 \mathrm{~mm}$ petri dishes and $50 \mu$ ldroplets of trypsin was placed in a petri dish and covered with mineral oil. Embryos $(n=10)$ were placed using glass pipette in the drops for one hour to allow partial zonal digestion. Digestion of the ZP was done under mineral oil in atmosphere at $37^{\circ} \mathrm{C}$ on the stage on inverted microscope. The measurement of $\mathrm{ZP}$ thickness was done using the microscope connected to computer software that allows to place mouse curser on the points of thickness to give readings.

\section{Embryo culturing}

After the AH techniques were completed, embryos were cultured in TCM-199 or Ham's F10. The embryos were cultured following different grouping methods in a $50 \mu \mathrm{l}$ droplets (one embryo in a drop, two embryos in a drop, three embryos in a drop and four embryos in a drop). This was aimed to determine if there is any effect of group embryo culture. The window period of 24 hours of culture was allowed for all embryos to undergo hatching after $\mathrm{AH}$ was performed. At the end of 24 hours of in vitro culture, the zonal thickness of all hatched embryos were measured and recorded.

\section{Embryo staining}

After 24 hours of embryo culture, the hatched blastocysts were stained with Hoechst 33258 . The hatched blastocysts were removed from the culture droplets and placed on a glass slide. The medium was aspirated using a micro-pipette to remove most of the volume of medium. The staining was done in the 4 -well plate where well one and well two were filled with $0.5 \mathrm{ml}$ of phosphate buffered saline and polyvinyl pyrrolidon medium, well three filled with $0.5 \mathrm{ml}$ fixative solution and well four filled with Hoechst 33258 of concentration $0.5 \mu \mathrm{g} / \mathrm{mL}$ in saline.

The hatched blastocyst-stage embryos were washed in well one and fixed with fixative solution (paraformaldehyde in D-PBS, 40 $\mathrm{mg} / \mathrm{ml}$ ) in well three for 30-60 minutes. After fixation in well three, embryos were stained in well four for a period of 20-30 minutes and washed again in well two before transferred to the slide. Stained embryos were placed on a new slide and labelled. A cover slip was placed to cover the embryo on the slide with Vaseline placed at each corner of the slip. The cover slip was used to cover the embryos to prevent foreign particles and spread the blastomeres.

Stain solution was added under the cover slip to cover the embryo. The stain is kept in the dark place at $2-8^{\circ} \mathrm{C}$ (SIGMA ALDRICH Co, Intervet South Africa). Freshly made stain was used once per day. The Cutex nail polish (M.A.C Studio Nail Lacquer) was used to cover all 
Table 1: Effect of assisted hatching techniques on blastocyst-stage embryos from mice.

\begin{tabular}{|c|c|c|c|}
\hline AH techniques & ZP thickness before AH $(\mu \mathrm{m})$ & Hatchability $(\%)$ & ZP thickness after culture $(\mu \mathrm{m})$ \\
\hline Control & $11.5 \pm 0.7^{\mathrm{b}}$ & $18.8 \pm 32.7^{\mathrm{b}}$ & $11.03 \pm 0.7^{\mathrm{b}}$ \\
\hline Laser & $11.6 \pm 1.2^{\mathrm{b}}$ & $51.8 \pm 40.0^{\mathrm{a}}$ & $11.27 \pm 1.2^{\mathrm{b}}$ \\
\hline Mechanical & $12.8 \pm 1.0^{\mathrm{a}}$ & $51.6 \pm 37.3^{\mathrm{a}}$ & $12.49 \pm 1.0^{\mathrm{a}}$ \\
\hline Enzymatic & $11.5 \pm 0.9^{\mathrm{b}}$ & $34.1 \pm 36.3^{\mathrm{ab}}$ & $7.31 \pm 1.4^{\mathrm{c}}$ \\
\hline Chemical & $11.5 \pm 0.8^{\mathrm{b}}$ & $33.6 \pm 36.9^{\mathrm{ab}}$ & $10.65 \pm 0.8^{\mathrm{b}}$ \\
\hline
\end{tabular}

${ }^{a-c}$ Different superscripts within the same column indicate significant differences among means $(P<0.05)$. AH: Assisted Hatching; ZP: Zona Pellucida.

Table 2: Effect of in vitro culturing media on blastocyst-stage embryo hatchability from mice.

\begin{tabular}{|c|c|c|c|}
\hline Culture media & ZP thickness before AH $(\mu \mathrm{m})$ & Hatchability $(\%)$ & ZP thickness after culture $(\mu \mathrm{m})$ \\
\hline Ham's F10 & $11.8 \pm 1.3^{\mathrm{a}}$ & $36.9 \pm 37.6^{\mathrm{a}}$ & $10.9 \pm 1.8^{\mathrm{a}}$ \\
\hline TCM-199 & $11.8 \pm 0.8^{\mathrm{a}}$ & $40.0 \pm 39.3^{\mathrm{a}}$ & $10.3 \pm 2.2^{\mathrm{b}}$ \\
\hline
\end{tabular}

a-bDifferent superscripts within the same column indicate significant differences among means $(\mathrm{P}<0.05), \mathrm{ZP}=$ zona pellucida.

Table 3: Effect of interaction between $\mathrm{AH}$ techniques and culture media on embryo hatching rate of mice.

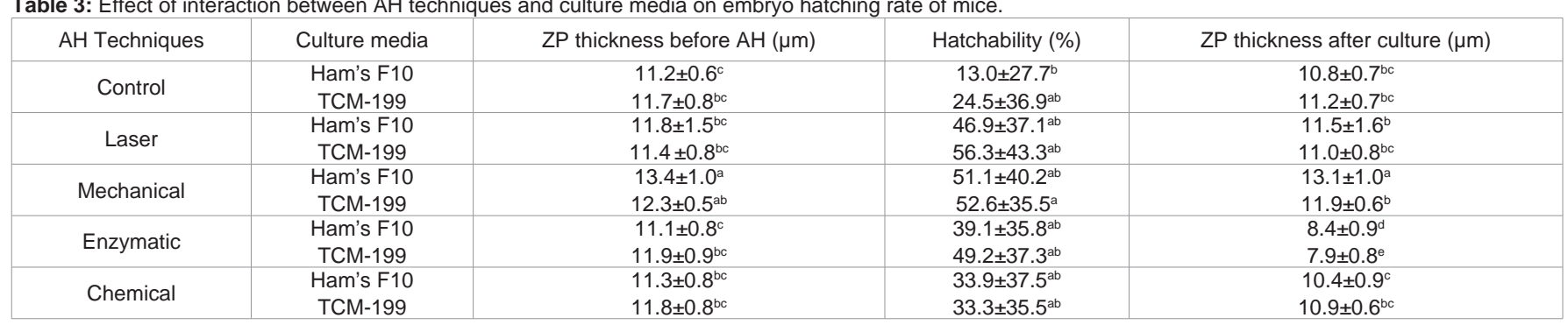

${ }^{a-e}$ Different superscripts within the same column indicate significant differences among means $(P<0.05)$.

AH: Assisted Hatching; ZP: Zona Pellucida, TCM-199: Tissue Culture Media 199.

the sides of the cover slip to prevent the hatched embryos to escape from underneath the slide. The labelled slide was turned upside down for counting the blastomeres under fluorescent UV light within 4 hours using an inverted microscope under X400 magnification (Nikon eclipse TI, Narishige co., Ltd. USA).

\section{Statistical analysis}

Data collected were subjected to analysis of variance using PROC General Linear Model of SAS version 9.4, where samples were randomly collected from the F1 generation mice. Where significant differences between groups were detected, the Tukey's test was used to separate the means and the following $\mathrm{p}$-values were used $(\mathrm{P}<0.05$; $\mathrm{P}>0.05$ and $\mathrm{P}<0.01)$.

\section{Ethical considerations}

The ethical clearance certificate referenced (SARDF/15/ ANS/03/0110) was obtained from the University of Venda Research Ethics Committee.

\section{Results}

The thickness of the $\mathrm{ZP}(7.3 \mu \mathrm{m})$ before $\mathrm{AH}$ and after culture for 24 hours $(12.8 \mu \mathrm{m})$ differed $(\mathrm{P}<0.01)$ (Table 1$)$. There was no different $(\mathrm{P}>0.05)$ on control group regarding the $\mathrm{ZP}$ thickness before and after culture. While other techniques showed difference and other showed no difference $(\mathrm{P}>0.05)$. Comparing the $\mathrm{AH}$ techniques, it was observed that they differed $(\mathrm{P}<0.05)$ in hatchability\% (Table 1).

The thickness of $\mathrm{ZP}$ ranged from 10.3 to $11.8 \mu \mathrm{m}$ both before $\mathrm{AH}$ and after in vitro culture (Table 2). The two in vitro culture media (TCM-199 or Ham's F10) did not differ $(\mathrm{P}>0.05)$ on hatching rate.
Both TCM-199 and Ham's F10 recorded 36.9 and 40.0 hatchability\%, respectively.

The interaction between $\mathrm{AH}$ techniques and in vitro culture media did not differ $(\mathrm{P}>0.05)$ on hatchability\% of blastocyst-stage embryos (Table 3). The highest hatchability percentage\% was with the laser AH techniques (56.3\%) cultured in TCM-199 medium followed by mechanical $\mathrm{AH}$ techniques (52.6\%) in the same in vitro culture medium. The lowest hatchability\% (33.3\%) was recorded in the chemical AH technique group. The thickness of the $\mathrm{ZP}$ was different before $\mathrm{AH}$ and after culture and ranged from 7.8 to $13.3 \mu \mathrm{m}(\mathrm{P}<0.01)$.

Considering the function of grouping embryos in different numbers per drop, there was a difference $(\mathrm{P}<0.01)$ observed on hatchability (Table 4 ). The hatchability increased with an increase number of embryos per drop until when the four embryos were grouped together and show slightly decline. The number of blastomeres counted after staining did not show any difference $(\mathrm{P}>0.05)$. The $\mathrm{ZP}$ thickness before and after in vitro culture did not differ $(\mathrm{P}>0.05)$.

Interaction of main factors (culture media, grouping of embryos per drop and $\mathrm{AH})$ did not differ $(\mathrm{P}>0.05)$ on the hatching percentage. The highest hatching percentage was observed with the use of laser AH techniques cultured in the TCM-199 culture medium and on the grouping of three embryos per drop with $(85.0 \%)$. The mechanical technique of AH using Ham's F10 with grouping of three embryos per drop yielded the second best hatchability (83.3\%). The third best hatching rate was attained from enzymatic method of $\mathrm{AH}$ using TCM-199 and three embryos per drop yielding (60.4\%). The lowest 
Table 4: Effect of number of embryos per drop on hatchability.

\begin{tabular}{|c|c|c|c|}
\hline Number of embryos per drop & ZP thickness before AH( $\mu \mathrm{m})$ & Hatchability $(\%)$ & ZP thickness after culture $(\mu \mathrm{m})$ \\
\hline 1 & $11.7 \pm 1.0^{\mathrm{a}}$ & $20.0 \pm 40.5^{\mathrm{c}}$ & $10.6 \pm 1.8^{\mathrm{a}}$ \\
2 & $11.7 \pm 1.1^{\mathrm{a}}$ & $28.7 \pm 29.7^{\mathrm{bc}}$ & $10.4 \pm 2.0^{\mathrm{a}}$ \\
3 & $11.8 \pm 1.1^{\mathrm{a}}$ & $59.1 \pm 38.8^{\mathrm{a}}$ & $71 \pm 9.1^{\mathrm{a}}$ \\
4 & $11.8 \pm 0.9^{\mathrm{a}}$ & $43.7 \pm 32.3^{\mathrm{ab}}$ & $10.5 \pm 2.1^{\mathrm{a}}$ \\
\hline
\end{tabular}

${ }^{a-c}$ Different superscripts within the same column indicate significant differences among means $(P<0.05)$.

ZP: Zona Pellucida.

Table 5: Interaction among AH techniques, culture media and number of embryos per drop.

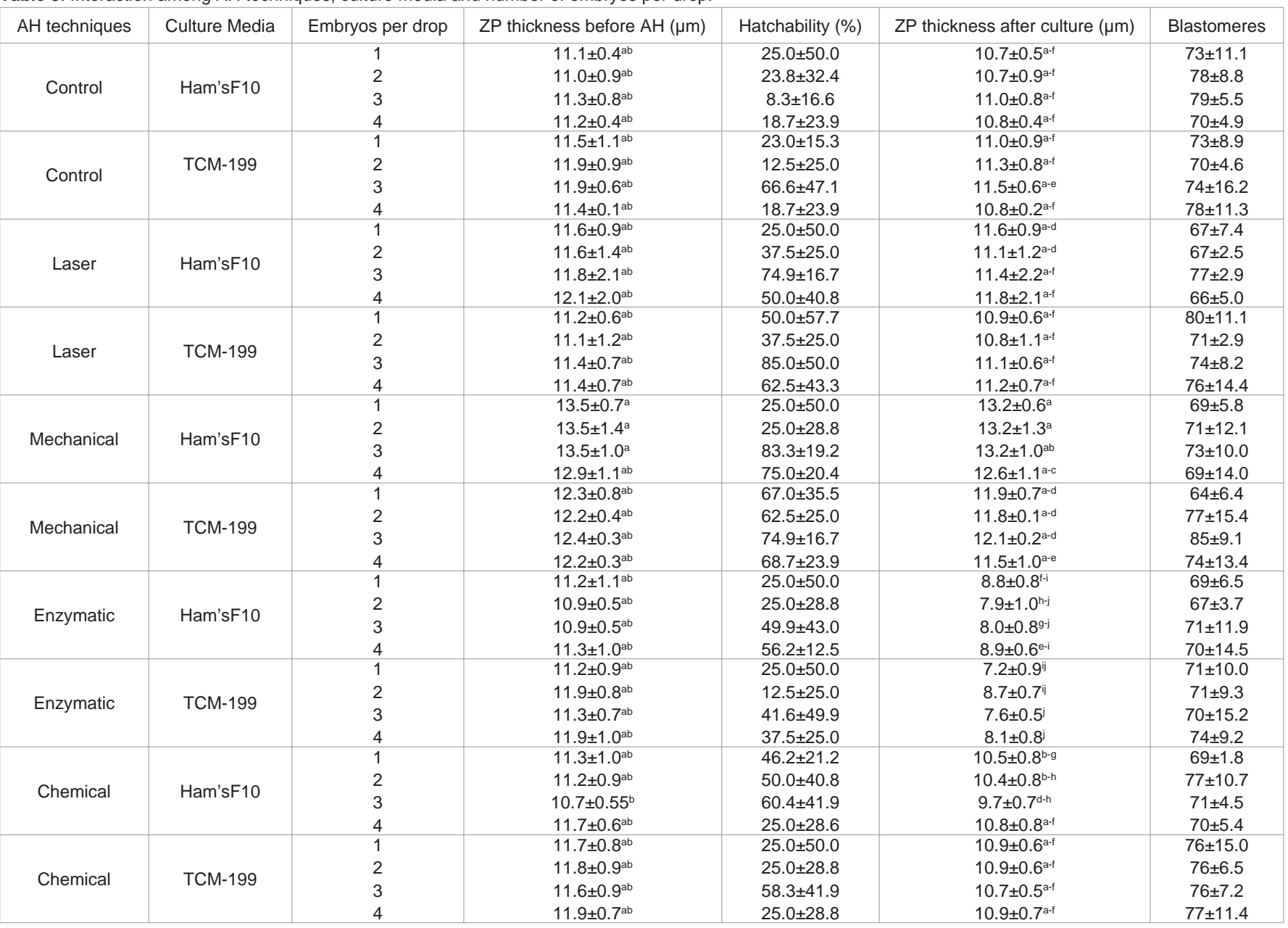

a-jDifferent superscripts within the same column indicate significant differences among means $(P<0.05)$.

AH: Assisted Hatching, ZP: Zona Pellucida; TCM-199: Tissue Culture Media 199.

hatchability in TCM-199 was observed when embryos are grouped into two per drop. The enzymatic AH, TCM-199 and grouping of three embryos per drop had the lowest effect on hatchability with (41.6\%). In all interactions of factors, hatchability increases with increase embryo grouping, but a slightly decline was observed when embryos are grouped into four per drop (Table 5).

\section{Discussion}

In the current study, blastocyst-stage embryos were subjected to four different $\mathrm{AH}$ techniques and in vitro cultured in both TCM199 and Ham's F10 for a period of 24 hours. The in vitro culture in TCM-199 and Ham's F10 media yielded 36.9 and 40.0\%, respectively. The results of this study indicated that there is a success hatchability of mice embryos in both Ham's F10 and TCM-199. The study also determined the thickness of the $\mathrm{ZP}$ before $\mathrm{AH}$ and after hatching.
The $\mathrm{ZP}$ before $\mathrm{AH}$ was used as a control measure to determine its thickness after $24 \mathrm{~h}$ of in vitro culture. The thickness of $\mathrm{ZP}$ determined in this study ranged from 10.8 to $13.6 \mu \mathrm{m}$. Moreover, prior to $\mathrm{AH}$, the thickness was observed to be different amongst the experimental mice and amongst embryos of the same individual. The difference in the thickness of the embryos within a column could be attributed to the fact that the mice used in the present study were of different ages, older than six weeks. This could be supported by the findings of [14] who reported that age of mice was one of the factors that determines the thickness of the ZP. [15] found that the thickness of the ZP plays a significant role in the hatching of mice embryos. A slight decrease in the thickness of the ZP was observed in non-assisted, mechanical, laser and chemical methods of $\mathrm{AH}$ with a significant decrease in the thickness observed in the enzymatic AH technique. 
The hatching percentages obtained from the present study for laser (33.6) and chemical techniques (51.8), were comparable to the findings of [16] who recorded a hatching percentage of (16.2) laser and (31.8) chemical. However, [17] found that the use of laser AH technique enhances complete hatching up to $80.0 \%$. From a study conducted by [15], they obtained up to 85.1 hatchability using mechanical AH technique. These results were higher than the results obtained from the present study and the difference in may be due to the difference in the time of in vitro culture used. The in vitro culture in the earlier study was carried out for 48 and 72 hours, whereas, in the present study the embryos were cultured for only 24 hours. [18] compared the mechanical and chemical AH techniques and has found higher hatchability in mechanical $\mathrm{AH}$. The reason was because the chemical AH technique tend to damage the inner cell mass due to the acidic solution. The study by [15], 77.6 hatching percentage was reported using the mechanical $\mathrm{AH}$ technique.

[19] reported that the volume of in vitro culture medium and density of embryos in a drop has a significant role in embryonic development, such that an increase in the density of embryos in a drop also increases embryonic hatching rate. This is probably by concentrating the trophic autocrine and paracrine factors [20]. In this study, culture of embryos in groups under a $50 \mu \mathrm{l}$ micro-droplet has a significant effect on the hatchability after 24 hours of culturing. The findings of this study do align with what other authors reported [21], because higher hatching was seen when embryos are cultured in groups than solely. [22] also agreed that the volume of in vitro medium also plays a significant role in the hatching of the murine embryos.

The interaction of $\mathrm{AH}$ techniques and in vitro culture media had a significant impact on hatching rate of embryos. In this study, it was found that the use of laser AH and TCM-199 yielded the highest results as compared to all other interactions. The in vitro culture of embryos without $\mathrm{AH}$ showed lower percentage of hatching when compared to $\mathrm{AH}$ techniques. The results show that the interaction between $\mathrm{AH}$ techniques and TCM-199 has a potential to initiate higher hatching percentages of mice blastocyst-stage embryos than in Ham's F10. Interaction of $\mathrm{AH}$ techniques, culture media and number of embryos per drop is represented on table 5. Although it is a complex study having three interactions, the results of these interactions seem to be the best overall on hatching. Enzymatic x TCM-199 x grouping of two embryos per drop yielded the lowest results of all interactions (12.5), while laser $\mathrm{x}$ TCM-199 $\mathrm{x}$ grouping of three embryos per drop yielded the best overall hatching (85.0). Hatchability\% (86.9) was obtained by [23] when embryos were grouped into nine in a $50 \mu$ l droplet. The results were slightly higher than that of the current study.

The variation of these results might be because only three embryos were grouped in the current study, which yielded (85.0) hatchability. Hatchability of (75.4) in AH was recorded by [23], with a slight decline to (70.8) when grouped in 12 embryos per drop. The same consistence of results was seen in this study with the grouping of four embryos per drop with a slight decline in hatchability to (75.0). Grouping of embryos into three per drop using Ham's F10 showed good hatching under mechanical, enzymatic and chemical techniques. Using TCM-199 and grouping of three embryos per drop yielded the highest hatching in the laser technique from the current study. [19] and [24-31] also supported that the density of incubation of mouse embryos do have beneficiary effect on hatching rate.

\section{Conclusion}

In conclusion, the results of the study indicated that the use of different $\mathrm{AH}$ techniques has got varying outcomes towards hatching rate, commonly when they are subjected to different in vitro culture media. Laser $\mathrm{AH}$ technique has higher hatchability as compared to all other AH techniques. It is then followed by mechanical, enzymatic and lastly chemical AH technique. Highest hatchability of embryos were recorded in TCM-199 as compared to in Ham's F10 medium. Furthermore, the embryo grouping during in vitro culture showed a great effect. Grouping of three embryos per droplet showed higher effect on hatchability as compared to other groupings (group of one, group of two and group of four embryos per drop). Interaction of $\mathrm{AH}$ techniques and in vitro culture media showed more effect on hatching rate. The $\mathrm{AH}$ techniques have the combined effects from in vitro culture media, the type of $\mathrm{AH}$ technique and the grouping of embryos per drop.

\section{Acknowledgement}

Authors would like to express thanks to University of Venda, University of Virginia and Centre of Excellence in Animal Assisted Reproduction. This study was registered as (SARDF/15/ANS/03/0110) in University of Venda Research. This study was supported by a grant from National Research Foundation (grant holder number 101974) and ETDP-SETA.

\section{Authors' Contributions}

Negota, Mphaphathi, Nethenzheni, Rammutla, Serota, Barry and Nedambale conceived the study, carried out the experiments, the study's design, coordinated and drafted the manuscript. Barry and Nedambale assisted with the interpretation of its results. All authors read and approved the final manuscript.

\section{References}

1. Park S, Kim H, Choi Y, Ahn K, Lee K, Yang J, et al. The effect of various assisted hatching techniques on the mouse early embryo development. Clin Exp Reprod Med. 2014; 41: 68-74.

2. Debrock S, Spiessens C, Peeraer K, De Loecker P, Willemen D, D'Hooghe TM. Higher implantation rate using modified quarter laser-assisted zona thinning in repeate implantation failure. Gynecol Obstet Invest. 2009; 67: 127-133.

3. Mathew A, Huai L, Avner H, Gerald M. A retroprospective study comparing three different assisted hatching techniques. Fertil Steril. 2009; 91: 13231325.

4. Choi D, An S, Park J, Kwon H, Lee S. Half assisted hatching significantly raise implantation rate in comparison with quarter assisted hatching. Fertil Steril. 2013; 100: 296-297.

5. Schimmel T, Cohen J, Saunders H, Alikani M. Laser-assisted zona pellucida thinning facilitates hatching and may disrupt the in vitro hatching process: A morpho kinetic study in the mouse. Hum Reprod. 2014; 29: 2670-2679.

6. Cohen J, Elsner $\mathrm{C}$, Kort $\mathrm{H}$. Impairment of hatching process following IVF in the human and improvement of implantation by assisted hatching using micromanipulation. Hum Reprod. 1990; 5: 7-13.

7. Das S, Blake D, Farquhar C, Seif MMW. Assisted hatching on assisted conception (IVF and ICSI). Cochrane Database Syst Rev. 2009; CD001894.

8. Wassarman PM. Zona pellucida glycoproteins. J Biol Chem. 2008; 283: 24285-24289. 
9. Montag M, Koll B, Holmes $\mathrm{P}$, Van Dee Ven $\mathrm{H}$. Significance of the number of embryonic cells and the state of the zona pellucida for hatching of mouse blastocysts in vitro versus in vivo. Biol Reprod. 2000; 62: 1738-1744.

10. Schiewe MC, Haze Leger NL, Sclimenti C, Balmacaan JP. Physiological characterization of blastocyst hatching mechanisms by use of a mouse antihatching model. Fertil Steril. 1995; 63: 288-294.

11. Murakami M, TOtoi NW, KOoka A, Tsuzuki. Effects of serum-free culture media on in vitro development of domestic Cat: Embryos following in vitro maturation and fertilization. Reprod Dom Anim. 2002; 37: 352-356.

12. Nedambale TL, Dinnyes A, Groen W. Comparison on in vitro fertilized bovine embryos cultured in KSOM or SOF and cryopreserved by slow freezing or vitrification. Theriogenology. 2004; 62: 437-449.

13. Mzezewa J, Misi T, Leon Van Rensburg D. Characterization of rainfall at semi-arid ecotope in the Limpopo province (South Africa) and its implications for sustainable crop production. Water SA. 2010; 1: 36.

14. Debra A, Gook DH, Edgar J, Borg M. Detection of zona pellucida proteins during human folliculogenesis. Hum Reprod. 2008; 23: 394-402.

15. Sung BP, Hye JK, Young BC, Kwang HA, Kee HL, Jung BY, et al. The effect of various assisted hatching techniques on the mouse early embryo development. Clin Exp Reprod Med. 2014; 41: 68-74.

16. Hsieh YY, Huang CC, Cheng TC, Chang CC, Tsai HD, Lee MS. Lase assisted hatching of embryos is better than the chemical method for enhancing the pregnancy rate in women with advanced age. Fertil Steril. 2002; 78: 179-182.

17. Neev J, Schiewe MC, sung VW, Kang ID, Berns MW, Tadir Y. Animal investigations. Assisted hatching in mouse embryos using a noncontact Ho:YSGG laser system. J Assist Reprod Genet. 1995; 12: 289-293.

18. Makrakis E, Angeli I, Agapitou K, Pappas K, Dafereras A, Pantsos K Laser versus mechanical assisted hatching: A prospective study of clinical outcomes. Fertil Steril. 2006; 86: 1596-1600.

19. Canseco RS, Sparks AE, Pearson RE, Gwazdauskas FC. Embryo density and medium volume effects on early murine embryo development. J Assis Reprod Gen. 1992; 9: 454-457.
20. Richter KS. The importance of growth factors for preimplantation embryo development and in-vitro culture. Obstetrics and Gynecology. 2008; 20: 292304.

21. Vutyavanich T, Saeng-Anan U, Sirisukkasem S, Piromlertamorn W. Effect of embryo density and microdrop volume on the blastocyst development of mouse two-cell embryos, Fertil Steril. 2011; 95: 1435-1439.

22. Kato $Y$, Tsunoda $Y$. Effects of the culture density of mouse zygotes on the development in vitro and in vivo. Theriogenology. 1994; 41: 1315-1322.

23. Shan JD, Chang LX, Jeffrey W, Ying PS, Ri CC. Effect of culture medium volume and embryo density on early mouse embryonic development: Tracking the development of the individual embryo. Published online: Springer Science Business Media. J Assist Reprod Gen. 2012; 29: 617-623.

24. Salahuddin S, Ookutsu S, Goto K. Effects of embryo density and co culture of unfertilized oocytes on embryonic development of in-vitro fertilized mouse embryos. Hum Reprod. 1995; 10: 2382-2385.

25. Downs SM, Hudson ED. Energy substrates and the completion of spontaneous meiotic maturation. Zygote. 2000; 8: 339-351.

26. Downs SM, Verhoeven. Glutamine and the maintenance of meiotic arrest in mouse oocytes: influence of culture medium, glucose and cumulus cells. Mol Reprod Develop. 2003; 66: 90-97.

27. Loutradis D, John D, Kiessling AA. Hypoxanthine causes a 2-cell block in random bred mouse embryos. Biol Reprod. 1997; 37: 311-316.

28. Movaghar B. "Expression of e-cadherin, leukemia inhibitory factor and progesterone receptor in mouse blastocysts after ovarian stimulation". Cell J. $2012 ; 14: 225-230$

29. Srisuparp S, Strakova Z, Fazleabas AT. "The role of chorionic gonadotropin (CG) in blastocyst implantation," Arc Med Res. 2001; 32: 627-634.

30. Teruel MT, Catalano RC, Cabodevila JA, Callejas SS. Regulation of embryo development by autocrine trophic factors. Biol Chem. 2005; 29: 183-186.

31. Yoshinaga Y. "Research on blastocyst implantation essential factors (BIEFs),"Am J Reprod Immunol. 2010; 63: 413-424. 